\title{
REVIEW OF APPLIED MATHEMATICAL MODELS FOR DESCRIBING THE BEHAVIOUR OF AQUEOUS HUMOR IN EYE STRUCTURES
}

\author{
M. DZIERKA* \\ WS-SPZOZ Zgorzelec, Opthalmological Ward \\ Lubańska St. 11-12, 59-900 Zgorzelec, POLAND \\ E-mail: martyna.dzierka@wp.pl \\ P. JURCZAK \\ University of Zielona Góra, Department of Mechanics \\ Szafrana St. 2, 65-516 Zielona Góra, POLAND \\ E-mail: P.Jurczak@ibem.uz.zgora.pl
}

\begin{abstract}
In the paper, currently used methods for modeling the flow of the aqueous humor through eye structures are presented. Then a computational model based on rheological models of Newtonian and non-Newtonian fluids is proposed. The proposed model may be used for modeling the flow of the aqueous humor through the trabecular meshwork. The trabecular meshwork is modeled as an array of rectilinear parallel capillary tubes. The flow of Newtonian and non-Newtonian fluids is considered. As a results of discussion mathematical equations of permeability of porous media and velocity of fluid flow through porous media have been received.
\end{abstract}

Key words: porous medium, Newtonian fluid, non-Newtonian fluid, coupe stress fluid, generalized second grade fluid, Darcy's rule, aqueous humor flow, trabecular meshwork, intraocular pressure, glaucoma.

\section{Introduction}

The aqueous humor is a substance responsible for keeping proper tension of the eyeball and for nutrition and purification of its structure. The correct value of intraocular pressure occurs when the aqueous humor is produced and drained into the ciliary body at the same rate the (Fig.1). As results of disorder of this process optic nerve can be damaged. Damage of the optic nerve causes permanent and total blindness. The value of intraocular pressure at $21 \mathrm{mmHg}$, considered as the highest acceptable value for healthy eye, was established (Niżankowska, 2006). A better understanding of pathomechanisms of diseases is the key to their effective treatment. Therefore it is very important to understand and describe the aqueous humor behavior in eye structures (Niżankowska, 2006).

In the literature a lot of mathematical models describing the behavior of the aqueous humor in the eye structures have been presented. All of them have in common the assignment of rheological properties of water to the aqueous humor. This is far-reaching simplification, because in aqueous collagen fibers, ascorbid acid and ions of various elements humor are supended. Therefore the aqueous humor should be treated as a couple stress fluid, or power series fluid (Walicka and Walicki, 1999).

The aqueous humor consists of $98 \%$ water. The composition of the aqueous humor is approximate to blood plasma composition. Differences of quantity in percents of suspended elements between that two fluids are very small. Therefore, a considerable approximation of the aqueous humor properties would be determine the rheological properties of blood plasma. Blood donation is a much less invasive procedure than aqueous humor donation. Blood donation procedure is also much less risky (Gabelt and Kaufman, 1995; Gedde, 2012).

Discussion on the flow of the aqueous humor in eye structures and its meaning for the control of intraocular pressure was initiated by Goldman. In 1950 he developed a technique for monitoring the direction of the flow and for making a quantitative analysis of the flow. Goldman assumed that the method of administration and sampling the dye did not affect the test results, and defined - from post-injection of the dye (fluorescein) into

\footnotetext{
* To whom correspondence should be addressed
} 
the structures of the eye dye - the velocity and flow directions of the fluid. The test method proposed by Goldman was very invasive, because the dye was administered directly by injection into the eyeball.

Currently used methods of flow testing are less invasive and based on fluoroopthometry. The dye is administered in the form of drops (Millar et al., 2011).

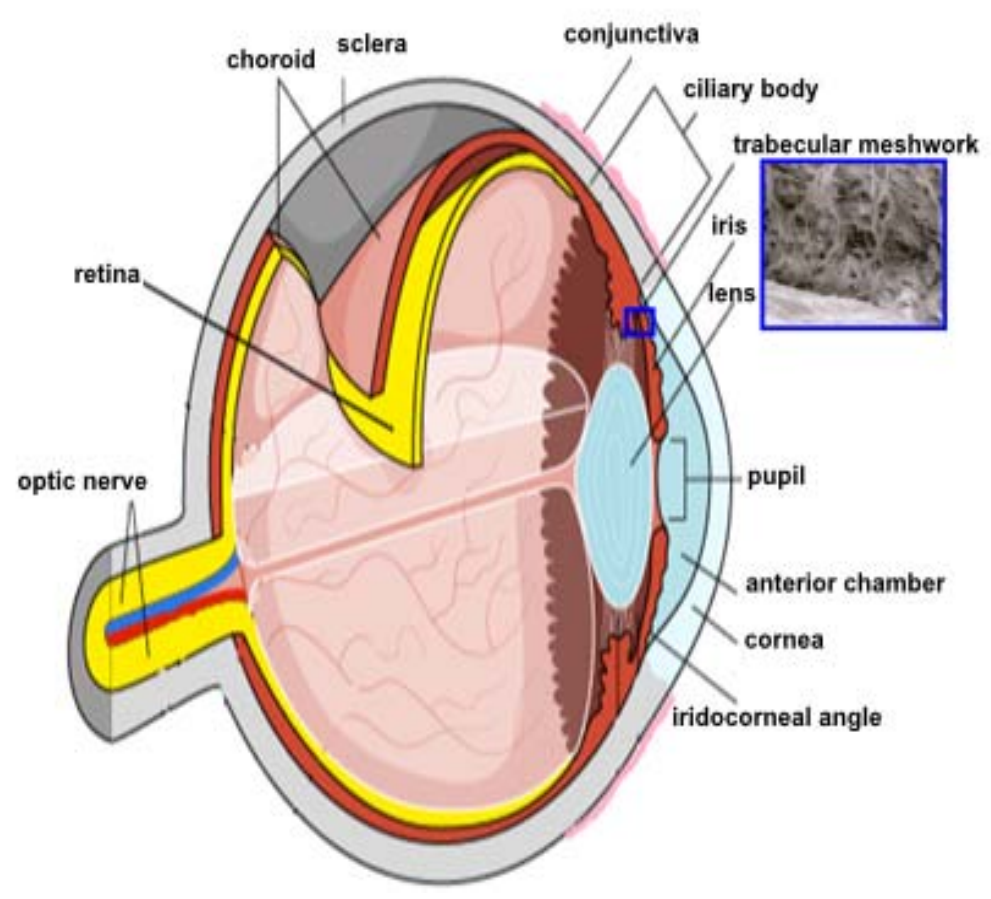

Fig.1. The structure of the eyeball.

Another group of studies are the studies that describe the physical properties of the aqueous humor and specify the flow rate produced in the ciliary body. These studies determine the effect of the amount of the fluid produced, and the pressure in the blood vessels of the IOP. Based on experimental methods methods of mathematical description of the behavior of the aqueous humor in the eye structures have been developed. These phenomena were mathematically described by Friedland (Heys et al., 2002). The model assumes a significant simplification, it is assumed that the tissues in the eye are rigid and do not affect the flow of the liquid. The geometry of the eye has also been simplified to the extent that it was possible to apply the Navier Stokes equations for the front and rear chamber. Friedland's model was developed by Tiedman (Heys et al., 2002). He introduced an iris as diaphragm pressure relief valve. He assumed that it is supported on the edges and bears pressure on the inner surface. He simplified the model assuming an infinitely small thickness of the iris and the lack of its effect on the flow of the aqueous humor. Heys's model constitutes a development of the two previous solutions and considers the effect of the iris of a finite thickness for the flow of the aqueous humor (Heys et al., 2002). Kaufman made certain assumptions about the passivity of the flow of the aqueous humor and its independence from energy. Moreover, he assumed that the outflow occurs only through the trabecular meshwork (Millar et al., 2011). The flow of the aqueous humor in the anterior chamber of the eye was analyzed by Fitt and Gonzalez (2006). All the models presented above and used to describe the behavior of the fluid within the eye have one common feature, namely the assumption that the rheological properties of the aqueous fluid are comparable to the properties of water.

The objective of this paper is to analyze the possibilities of using rheological models of Newtonian and non-Newtonian fluids for mathematical modeling of the aqueous humor flow in the trabecular meshwork. The trabecular meshwork is modeled as a porous medium, which is a rectilinear array of parallel capillary tubes. A Newtonian fluid, a couple stress fluid and a generalized second grade fluid of a Shulman's type were considered as examples. 


\section{Mathematical models of the aqueous humor flow}

\subsection{Kaufman's model}

The behaviour of the aqueous humor in the eyeball according to Kaufman and coworkers (Gabelt and Kauffman, 1995; Millar et al.,2011) can be presented by the following equations

$$
\begin{aligned}
& F_{i}=F_{s}+F_{f}, \\
& F_{o}=F_{t}+F_{u}, \\
& C_{t o t}=C_{t r a b}+C_{u}+C_{p s}, \\
& F=F_{i}=F_{o}
\end{aligned}
$$

where : $F$ - the flow rate, $F_{i}$ - the total flow of the aqueous humor to the eye, $F_{s}$ - the flow of the aqueous humor through active secretion, $F_{f}$ - the flow of the aqueous humor by filtration, $F_{o}$ - the total outflow of the aqueous humor from the eye, $F_{t}$ - the outflow of the aqueous humor through the trabecular meshwork, $F_{u}$ - the outflow of the aqueous humor through the sclera, $C_{t o t}, C_{p p}$-total conductance of the flow, $C_{t r a b}$, $C_{T M}$ - conductance of the flow through the trabecular mesh, $C_{u}$ - conductance of the flow through the sclera, $C_{p s}$ - conductance of inflow of the aqueous humor.

The flow of the aqueous humor in the eye may be presented by the hydraulic model and Goldman's equation, assuming that the liquid flow is passive, an energy-independent and occurs in the direction of the pressure gradient. In addition, it is assumed that the outflow occurs only through the trabecular meshwork. This simplification shall be allowed, because $90 \%$ of the aqueous humor flows in this way. It can then be assumed that Eqs (2.2) and (2.3) determining the pressure difference $\Delta p$ are true (Millar et al., 2011)

$$
\Delta p=p_{i}-p_{v}
$$

and the flow rate $F$

$$
F=C_{\text {trab }}\left(p_{i}-p_{v}\right)
$$

where $p_{i}$ - the intraocular pressure, $p_{v}$ - the episcleral vessels pressure.

For a human body the flow rate $F$ amounts to

$$
F=0.28\left[\frac{\frac{\mu l}{\mathrm{~min}}}{\mathrm{mmHg}}\right](15.6[\mathrm{mmHg}]-7.6[\mathrm{mmHg}])=2.24\left[\frac{\mu l}{\mathrm{~min}}\right] .
$$

\subsection{Heys's model}

Heys's model (Heys et al., 2002) assumes that the aqueous humor has the following properties: density $1000\left[\mathrm{~kg} / \mathrm{m}^{3}\right]$, viscosity $7.5 \cdot 10^{-4}[\mathrm{~kg} / \mathrm{ms}]$ for $37\left[{ }^{\circ} \mathrm{C}\right]$, the Reynolds constant of 0.001 was determined for the flow in the eyeball by Friedman. The length scale of the flow of the aqueous humor was 
$5 \cdot 10^{-4}[\mathrm{~m}]$, and the characteristic velocity of the flow was $2 \cdot 10^{-6}\left[\frac{\mathrm{m}}{\mathrm{s}}\right]$. Heys's model did not consider the dependence between the flow rate and intraocular pressure, his model also ignored the effects of the mass forces (Heys et al., 2002). Equations determining the motion of a viscous liquid can be presented as

$$
\begin{aligned}
& \nabla \underline{v}=0, \\
& \rho \frac{d \underline{v}}{d t}=-\nabla P+\mu \nabla^{2} \underline{v},
\end{aligned}
$$

leaving aside in Eqs (2.4) the effect of inertial forces we obtain (Heys et al., 2002)

$$
\begin{aligned}
& \nabla \underline{u}=0, \\
& -\nabla P+G \nabla^{2} \underline{u}=0 .
\end{aligned}
$$

The geometrical dimensions of the eye in the model were taken from ultrasound and biomicroscopic examinations. The model takes into account the phenomenon of changing the shape of the iris and blinking. The eye model was created and analyzed using the finite element method in the software module FIDAP FIGEN. Heys gave the dependence that determines the flow of the aqueous humor within the eye of additional parameters (2.6), and a simplified version (2.7) (Heys et al., 2002)

$$
\begin{aligned}
& v_{n}=\frac{C_{T M}\left(p_{o}-p_{v}\right)+F_{U}}{A_{o}}, \\
& v_{n}=\frac{C_{P P}\left(p_{o}-p_{v}\right)}{A_{v}}
\end{aligned}
$$

where $p_{o}$ - the pressure measured at the outlet of the trabecular meshwork, $p_{v}-$ the episcleral vessels pressure.

The simplified version excludes the impact from drainage of the aqueous humor through the sclera $\left(F_{U}\right)$ for a normal flow rate of fluid $\left(v_{n}\right)$. It was assumed that the aqueous humor flows only through the trabecular network and therefore the conductance of the flow through the structure $\left(C_{T M}\right)$ was replaced by the total conductance $\left(C_{P P}\right)$. The flow area of the trabecular meshwork $\left(A_{o}\right)$ was replaced by the total contact surface of the vitreous and aqueous humor $\left(A_{v}\right)$.

\subsection{Fitt's-Gonzalez's model}

flow.

Fitt and Gonzalez (2006) analyzed the flow of fluid for five different groups of factors forcing the

The factors were divided into groups associated with:

- fluctuations of temperature between individual structures of the eye.

- the production of the aqueous humor in the ciliary body,

- changes in eye position,

- effects of accommodation,

- eye movements during REM sleep.

They developed equations that describe the flow of the aqueous humor in the eye structures, by using the finite element method implemented in Femlab software (Fitt and Gonzalez, 2006) 


$$
\begin{aligned}
& -\frac{p_{x}}{\rho_{0}}+v u_{z z}+g\left[1-\alpha\left(T-T_{0}\right)\right]=0, \\
& -\frac{p_{y}}{\rho_{0}}+v v_{z z}=0 \\
& p_{z}=0 \\
& u_{x}+v_{y}+w_{z}=0 \\
& T_{z z}=0 .
\end{aligned}
$$

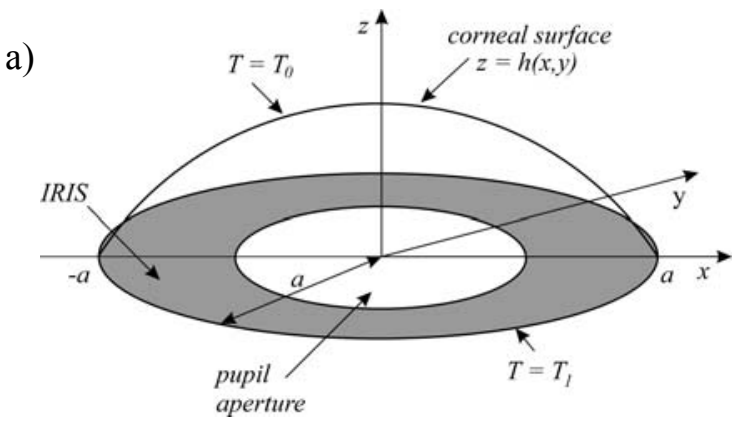

b)

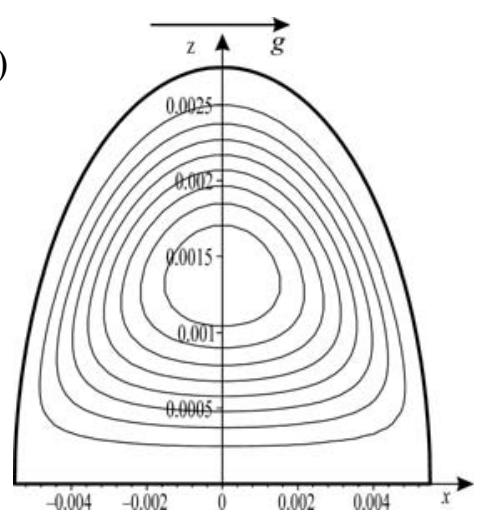

d)

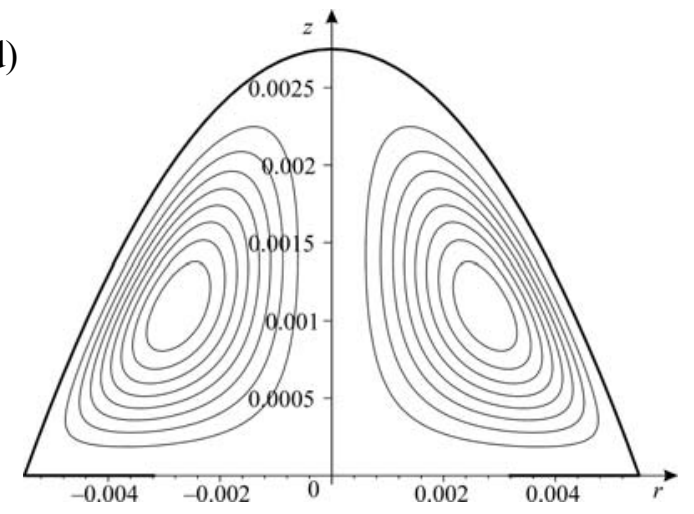

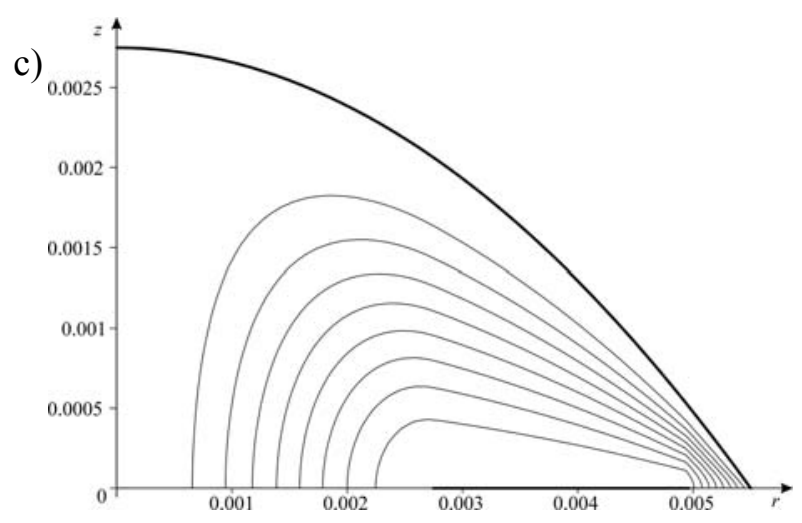

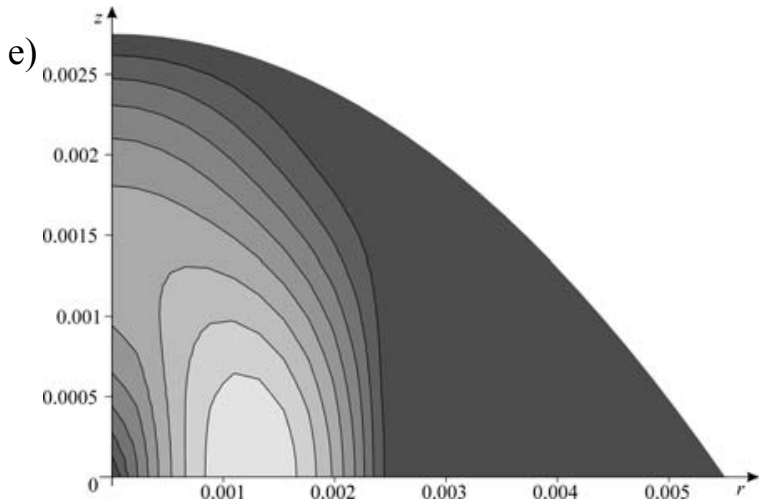

Fig.2. Aqueous humor flow in Fitt's-Gonzalez's model: a) boundary conditions, b) flow forced by temperature differences, c) flow forced by production of the aqueous humor by the ciliary body, d) flow during sleep, e) flow forced by eyeball movements (Fitt and Gonzalez , 2006). 
The problem was analyzed considering the following boundary conditions

$$
\begin{aligned}
& u=v=0, \quad w=w_{0}(x, t), \quad T=T_{1} \quad \text { dla } \quad z=0, \\
& u=v=w=0, \quad T=T_{0} \quad \text { dla } \quad z=h(x, y) .
\end{aligned}
$$

Assuming the simplest case (i.e., no transfer through the aperture of the pupil $\left(w_{0}=0\right)$, the gravity acting along the positive axis, and hydrostatic pressure), we determine $v=0$, therefore the flow is in a vertical plane $(x, z)$ of the front chamber. The solution for this case is given by the following relationships

$$
\begin{gathered}
p=p_{a}+\frac{g \rho_{0}(x+a)\left(2-\alpha\left(T_{1}-T_{0}\right)\right)}{2}, \\
T=T_{1}+\frac{z\left(T_{0}-T_{1}\right)}{h}, \\
\psi=-\frac{\left(T_{1}-T_{0}\right) g a z^{2}(z-h)^{2}}{24 v h} .
\end{gathered}
$$

In Eqs (2.8)-(2.17) fluid velocity is determined by $\boldsymbol{q}=(u, v, w)$, the coordinate system is shown in Fig.2, where $p$ means the pressure, $p_{a}$-environmental pressure, $g$-acceleration due to gravity, $a$ - the radius of the eyeball, $\rho_{0}$ - density, $\alpha$ - the coefficient of linear expansion, $T$ - temperature, $T_{0}, T_{1}-$ the temperature of the front and rear part of the cornea, $v$ - kinematic viscosity, $\psi$ - current function.

Based on the analysis of the results obtained it was found that a temperature difference between the eye structures has the greatest impact on the circulation of the fluid in the structures of the eye. The velocity of the aqueous humor thus obtained is an order of magnitude greater than the flow rate enforced by other factors.

\section{The proposed computational models for the flow of the aqueous humor through the trabecular meshwork}

Most of the aqueous humor is drained from the eye to the ciliary body through the trabecular meshwork. In this way, about $90 \%$ of the aqueous humor is discharged. That is why it is necessary to develop computational models allowing a description of the behavior of biological fluids in the tissues. To approximate the behavior of the fluid within the trabecular network a model of a porous medium can be used, which consists of an array of parallel and straight glass capillary tubes, arranged parallel to the direction of the flow. In contrast, in mathematical modeling of the aqueous humor models of a Newtonian fluid, a couple-stress fluid and Shulman's fluid can be used.

\subsection{Flow of a Newtonian fluid through a porous medium}

The generalized model of a Newtonian fluid flow in porous media - obtained from Navier and Stokes - is known in the literature as a Brinkman-Forchheimer-Darcy model (Amiri and Vafai, 1994; 1998; Nakayama et al., 2004; 2006). The equation of motion for this model can be represented by the following relationships (Walicka, 2002; Walicka and Walicki, 2012): 
- continuity equation

$$
\nabla \bar{v}=0
$$

- momentum equation

$$
\frac{\rho}{\varphi_{p}}\left[\frac{\partial \overline{\mathbf{v}}}{\partial t}+(\overline{\mathrm{v}} \nabla) \overline{\mathbf{v}}\right]=-\nabla p+\frac{\mu}{\varphi_{p}} \nabla^{2} \overline{\mathbf{v}}-\frac{\mu}{\Phi_{n}} \overline{\mathbf{v}}-\frac{\rho \boldsymbol{F} \varphi_{p}}{\sqrt{\Phi_{n}}}|\overline{\mathbf{v}}| \overline{\mathbf{v}}
$$

where $\varphi_{p}$ is the medium porosity, $\Phi_{n}$ - permeability, $\mu$ - dynamic viscosity, $\boldsymbol{F}$ - geometric tensor, $\overline{\mathrm{v}}-$ averaged velocity, $\bar{p}$ - averaged pressure.

Consider a Newtonian fluid in a porous layer having a thickness $H_{p}$, shown in Fig.3, which is a matrix composed of parallel rectilinear tubes. The flow rate of a Newtonian fluid in that layer - in accordance with Darcy's law - is equal to Walicka (2002)

$$
\overline{\mathbf{v}}=-\frac{\Phi_{n}}{\mu} \frac{d \bar{p}}{d y}
$$

where $\Phi_{n}$ is permeability of the porous layer.

The flow rate of a Newtonian fluid in the capillary tube having a radius $r_{c}$ (Fig.3) is given by the equation (Walicka, 2012)

$$
\mathrm{v}_{y}=\left(1-\frac{r^{2}}{r_{c}^{2}}\right)\left(-\frac{d \bar{p}}{d y}\right)
$$

and the flow rate $\bar{Q}$ is determined by the equation

$$
\bar{Q}=2 \pi \int_{0}^{r_{c}} v_{y} r d r
$$

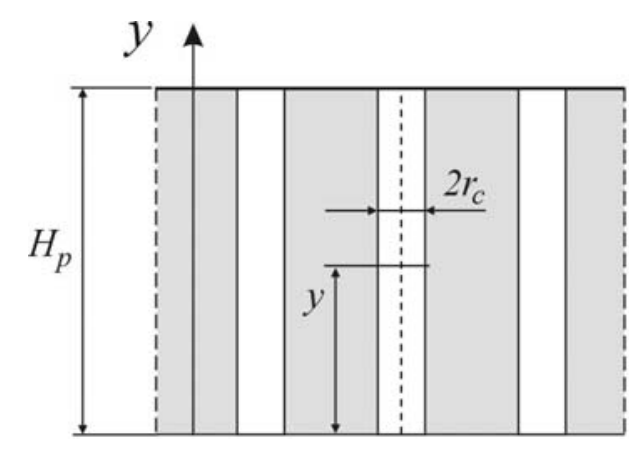

Fig.3. Porous area composed of parallel rectilinear tubes.

Finally, the Newtonian fluid flow rate in the capillary tube - basing on formulas (3.4) and (3.5) -is given by the equation 


$$
\bar{Q}=2 \pi \int_{0}^{r_{c}}\left(1-\frac{r^{2}}{r_{c}^{2}}\right)\left(-\frac{d \bar{p}}{d y}\right) r d y=-\frac{\pi r_{c}^{4}}{8 \mu} \frac{d \bar{p}}{d y},
$$

and averaged velocity in the capillary tube is equal to

$$
\overline{\mathrm{v}}_{a}=\frac{\bar{Q}}{\pi r_{c}^{2}}=-\frac{r_{c}^{2}}{8 \mu} \frac{d \bar{p}}{d y} .
$$

Taking into account the porosity of the medium consisting of capillary tubes

$$
\varphi_{p}=\frac{N_{c} \pi r_{c}^{2} H_{p}}{A H_{p}}=\frac{N_{c} \pi r_{c}^{2}}{A}
$$

where $N_{c}$ is the number of capillary tubes on the surface $A$ of the porous medium, we obtain

$$
\bar{v}=\bar{v}_{a} \varphi_{p}=-\frac{r_{c}^{2} \varphi_{p}}{8 \mu} \frac{d \bar{p}}{d y}
$$

Comparing Eqs (3.3) and (3.9), we get (Walicka, 2002; 2012; Walicka and Walicki, 2012)

$$
\Phi_{n}=\frac{r_{c}^{2} \varphi_{p}}{8}
$$

the equation determining permeability of the porous medium for a Newtonian fluid.

\subsection{Flow of couple-stress fluid} researchers.

Flows of couple-stress fluids were investigated first by Stokes (1966) and other subsequent

Considering the analysis presented in the papers (Walicka, 2002; 2012; Walicka and Walicki, 1999; 2012) equations of motion of couple-stress fluids may be presented in the following form

$$
\begin{aligned}
& \operatorname{div} \mathbf{v}=0 \\
& \rho \frac{d \mathbf{v}}{d t}=\rho \boldsymbol{f}+\rho \frac{1}{2} \operatorname{curl} \boldsymbol{l}-\operatorname{grad} p+\mu \nabla^{2} \mathbf{v}-\eta \nabla^{4} \mathbf{v}, \\
& \rho \frac{d e}{d t}=\operatorname{div}(\lambda \operatorname{grad} T)+2 \mu \boldsymbol{D}: \boldsymbol{D}+4 \eta \boldsymbol{K}: \boldsymbol{K}, \\
& \boldsymbol{K}=(\operatorname{grad} \boldsymbol{\omega})^{T}, \quad \boldsymbol{\omega}=\frac{1}{2} \operatorname{curl} \mathbf{v} .
\end{aligned}
$$

In the theory of couple-stress fluids two tensors exist:

- the stress tensor 


$$
\boldsymbol{T}=-p \boldsymbol{I}+2 \mu \boldsymbol{D}-2 \eta \nabla^{2} \boldsymbol{W}
$$

where $\boldsymbol{D}$ is a symmetric $\operatorname{grad} \mathbf{v}$ and $\boldsymbol{W}$ is an asymmetric part of the tensor

$$
\begin{gathered}
\boldsymbol{D}=\frac{1}{2}\left(\boldsymbol{L}+\boldsymbol{L}^{T}\right)=\frac{1}{2}\left(\nabla \mathbf{v}+\nabla \mathbf{v}^{T}\right), \quad \boldsymbol{D}=\boldsymbol{D}^{T}, \\
\boldsymbol{W}=\frac{1}{2}\left(\boldsymbol{L}-\boldsymbol{L}^{T}\right)=\frac{1}{2}\left(\nabla \mathbf{v}-\nabla \mathbf{v}^{T}\right), \quad \boldsymbol{W}=-\boldsymbol{W}^{T},
\end{gathered}
$$

$\boldsymbol{D}$ is the deformation rate tensor, $\boldsymbol{W}$ is the spin tensor, symbol ${ }^{T}$ is the tensor matrix transposition

$$
\operatorname{grad} \mathbf{v}=\nabla \mathbf{v}
$$

- the couple-stress tensor

$$
\boldsymbol{M}_{s}=4 \eta \boldsymbol{K}+4 \eta^{\prime} \boldsymbol{K}^{T}
$$

where $\eta$ and $\eta^{\prime}$ are material constants associated with couple-stress.

Consider the flow of a couple-stress fluid through the capillary having a radius $r_{c}$, as shown in Fig.4. The axis $y$ is the axis of symmetry of the tube. The velocity field is described by the equation

$$
v_{y}=\left\{1-\frac{r^{2}}{r_{c}^{2}}-\frac{2\left(\eta-\eta^{\prime}\right)\left[I_{0}\left(\frac{r_{c}}{l}\right)-I_{0}\left(\frac{r}{l}\right)\right]}{\frac{r_{c}}{l}\left[\eta \frac{r_{c}}{l} I_{0}\left(\frac{r_{c}}{l}\right)-\left(\eta+\eta^{\prime}\right) I_{1}\left(\frac{r_{c}}{l}\right)\right]}\right\} \frac{r_{c}^{2}}{4 \mu}\left(-\frac{d \bar{p}}{d y}\right)
$$

where $I_{0}(\rho)$ and $I_{l}(\rho)$ are the modified Bessel functions of the zeroth and the first order and the first kind.

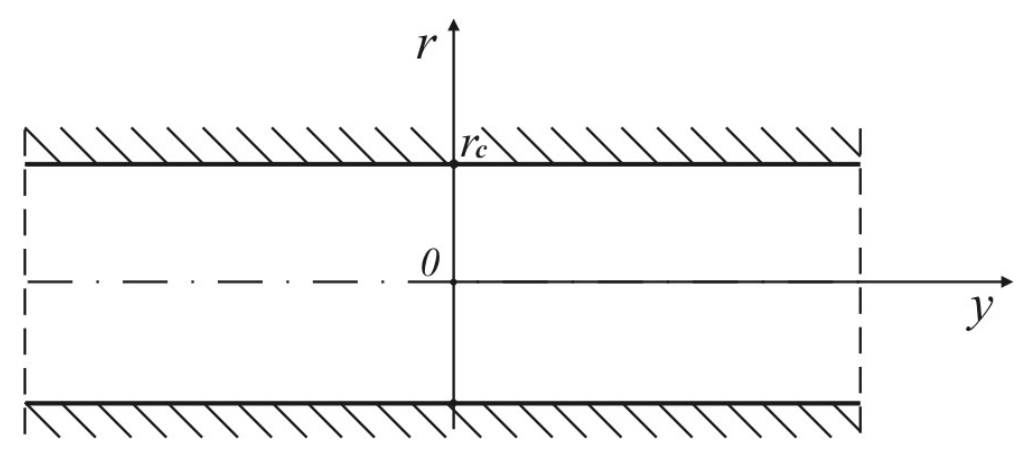

Fig.4. The geometry of the capillary tube.

The flow rate $\bar{Q}$ is defined by Eq.(3.5). Substituting Eq.(3.19) into Eq.(3.5), we get 


$$
\bar{Q}=\frac{\pi r_{c}^{4} \Phi_{l}(\Psi)}{8 \mu}\left(-\frac{d \bar{p}}{d y}\right)
$$

where

$$
\begin{aligned}
& \Phi_{l}(\Psi)=1-\frac{4\left(\eta-\eta^{\prime}\right)}{N(\Psi)}\left[I_{0}(\Psi)-\frac{2}{\Psi} I_{l}(\Psi)\right], \quad \Psi=\frac{r_{c}}{l} \approx 1, \\
& N(\Psi)=\Psi\left[\eta \Psi I_{0}(\Psi)-\left(\eta+\eta^{\prime}\right) I_{l}(\Psi)\right], \quad l^{2}=\frac{\eta}{\mu} .
\end{aligned}
$$

Then we obtain

$$
\overline{\mathrm{v}}_{a}=\frac{\bar{Q}}{\pi r_{c}^{2}}=\frac{r_{c}^{2}}{8 \mu} \Phi_{l}(\Psi)\left(-\frac{d \bar{p}}{d y}\right), \quad \text { for } \quad \eta=\eta^{\prime} \quad \Phi_{l}=1-\beta_{c} ; \quad \beta_{c}=8\left(\eta-\eta^{\prime}\right)^{2}
$$

and

$$
\Phi_{c}=\frac{r_{c}^{2} \varphi_{p}}{8} \Phi_{l}(\Psi),
$$

Is the equation describing permeability of a couple-stress fluid.

\subsection{Flow of generalized second grade fluids}

Man and Sun (1987) and Tsai et al. (1988) were the first who presented the results of research on the flow of generalized second grade fluids. Results of research on the flow of the aforementioned fluids can also be found in the papers by Walicki and Walicka (Walicka 2002a; 2002b; Walicki and Walicka 1998; 1999; 2000a; 2000b; 2000c).

Motion equations of generalized second grade fluids can be presented in the form of

$$
\begin{aligned}
& \operatorname{div} \mathbf{v}=0, \\
& \rho \frac{d \mathbf{v}}{d t}=\rho \boldsymbol{f}+\operatorname{div} \boldsymbol{T}
\end{aligned}
$$

where $\boldsymbol{T}$ depends on the fluid model. Let us consider two models of fluids - proposed by Walickis (Walicki and Walicka, 1998) - based on the non-linear Shulman's model. Constitutive equations can then be presented as:

- model I

$$
\boldsymbol{T}=-p \mathbf{1}+M \boldsymbol{A}_{1}+\alpha_{1} \boldsymbol{A}_{1}^{2}+\beta_{1} \boldsymbol{A}_{2},
$$

- model II

$$
\boldsymbol{T}=-p \boldsymbol{I}+M\left(\boldsymbol{A}_{1}+\alpha_{2} \boldsymbol{A}_{1}^{2}+\beta_{2} \boldsymbol{A}_{2}\right)
$$


where

$$
M=\left[\tau_{0}^{\frac{1}{n}}+(\mu A)_{m}^{\frac{1}{m}}\right]^{n} A^{-1}, \quad A=\left[\frac{1}{2} \operatorname{tr}\left(A_{1}^{2}\right)\right]^{\frac{1}{2}}
$$

where $\boldsymbol{A}_{\boldsymbol{1}}$ and $\boldsymbol{A}_{2}$ are first two Rivlin's-Ericksen's tensors defined by the equations

$$
\boldsymbol{A}_{l}=2 \boldsymbol{D}=\left(\nabla \mathbf{v}+\nabla \mathbf{v}^{T}\right), \quad \boldsymbol{A}_{2}=\dot{\boldsymbol{A}}_{l}+\boldsymbol{A}_{l} \nabla \mathbf{v}+\nabla \mathbf{v}^{T} \boldsymbol{A}_{l} .
$$

Consider the steady laminar flow of a generalized second grade fluid in the capillary tube having a radius $r_{c}$ (Fig.5).

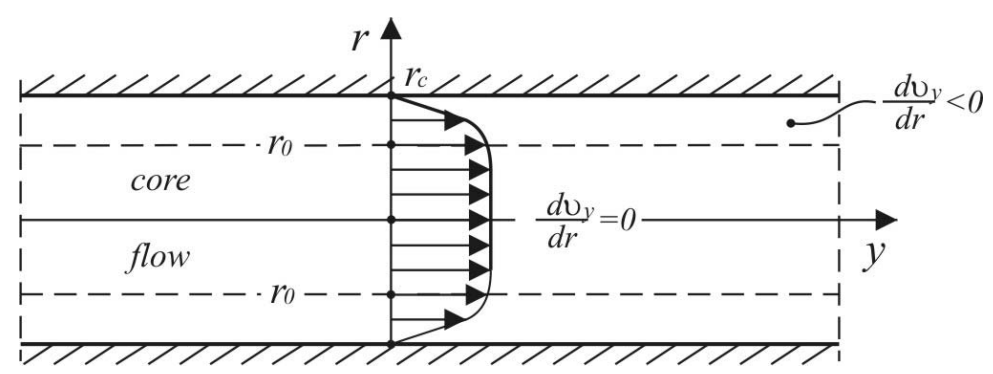

Fig.5. The coordinate system and velocity field in the capillary tube.

The velocity field is defined as follows:

- for shear flow

$$
\mathrm{v}_{y s}(r)=\frac{r_{0}}{\mu}\left(\frac{r_{0} \tau_{0}}{r_{c}}\right)^{\frac{m}{n}} Y^{\frac{m}{n}} \sum_{i=0}^{m} \Phi_{i}\left[1-\left(\frac{r}{r_{c}}\right)^{\frac{m+n-i}{n}}\right],
$$

- for core flow

$$
\mathrm{v}_{y c}=\mathrm{v}_{y s}\left(r_{0}\right)
$$

where

$$
\Phi_{i}=(-1)^{i} \frac{n}{m+n-i} C_{m}^{i} Y^{\frac{m+n-i}{n}} \quad \text { and } \quad Y=-\frac{r_{c}}{2 \tau_{0}} \frac{\partial \bar{p}}{\partial y}
$$

The flow rate $\bar{Q}$ is given by the equation 


$$
\bar{Q}=2 \pi \int_{0}^{r_{c}} \mathrm{v}_{y} r d r=2 \pi\left[\int_{0}^{r_{0}} \mathrm{v}_{y c} r d r+\int_{r_{0}}^{r_{c}} \mathrm{v}_{y s} r d r\right]
$$

Using Eqs (3.32) and (3.33), we obtain

$$
\bar{Q}=\frac{\pi r_{c}^{3} \tau_{0}^{\frac{m}{n}}}{\mu} Y^{-3} \sum_{i=0}^{m}(-1)^{i} \frac{n}{m+3 n-i} C_{m}^{i}\left[Y^{\frac{m+3 n-i}{n}}-1\right]
$$

Consider at the beginning the flow of a generalized second grade fluid of a power-law type in porous media consisting of a parallel system of straight capillaries. Equation (3.34) describes the flow rate in the capillary tube

$$
\bar{Q}=\frac{\pi r_{c}^{m+3}}{2^{m}(m+3) \mu}\left(-\frac{d \bar{p}}{d y}\right)^{m}
$$

while the average speed in the capillary tube is given as follows

$$
\overline{\mathrm{v}}_{a}=\frac{\bar{Q}}{\pi r_{c}^{2}}=\frac{r_{c}^{m+3}}{2^{m}(m+3) \mu}\left(-\frac{d \bar{p}}{d y}\right)^{m} .
$$

The analogue of Darcy's law can be represented by the equation

$$
\overline{\mathrm{v}}=\overline{\mathrm{v}}_{a} \varphi_{p}=\frac{r_{c}^{m+3} \varphi_{p}}{2^{m}(m+3) \mu}\left(-\frac{d \bar{p}}{d y}\right)^{m}
$$

or

$$
\bar{v}=-\frac{\Phi_{p}}{\mu}\left(-\frac{d \bar{p}}{d y}\right)^{m}, \quad \Phi_{p}=\frac{r_{c}^{m+1} \varphi_{p}}{2^{m}(m+3)}
$$

It can be noticed that the analogue of Darcy's law has the same form (independent of coefficients describing the viscoelastic properties of material $\alpha_{i}$ and $\beta_{i}$ ) for both models of generalized second grade fluid of a power-law type; the flow of Rivlin's-Ericksen's fluid (viscoelastic fluid) to the porous layer is defined by Darcy's law given by Eq.(3.3). Following in a similar way, an analogue of Darcy's law for any model of fluid can be designated.

Consider the flow of a Shulman's generalized second grade fluid of a power-law type in a porous medium consisting of a system of parallel, straight capillaries. Introducing the following equation

$$
Y=\frac{r_{0}}{r_{c}}, \quad \tau_{0}=\frac{r_{c} Y}{2}\left(-\frac{d \bar{p}}{d y}\right),
$$

we obtain - considering Eqs (3.39) and (3.34) - equations for the Shulman's type fluid flow velocity through a porous layer. Results for selected empirical models of viscoplastic fluids are presented in Tab.1 (Walicka, 2012) 
Table 1. The mathematical models of viscoplastic fluids (Walicka, 2002).

\begin{tabular}{|c|c|c|c|}
\hline $\begin{array}{c}\text { Model's inventor } \\
\text { name }\end{array}$ & Mathematical Form & Designation & Equation no. \\
\hline Bingham & $\tau=\tau_{0}+\mu \dot{\gamma}$ & \multirow{7}{*}{$\begin{array}{c}\tau \text { - shear stress, } \\
\tau_{0} \text { - yield point, } \\
\dot{\gamma} \text { - shear rate, } \\
\mu \text { - shear or plastic viscosity } \\
\mu_{0}, \mu_{\infty} \text { - boundary viscosity } \\
m, n \text { - exponents of the } \\
\text { rheological equations of } \\
\text { state } \\
\kappa \text { - material constant }\end{array}$} & $(3.40)$ \\
\hline Herschel-Bulkley & $\tau=\tau_{0}+(\mu \dot{\gamma})^{\frac{l}{m}}$ & & $(3.41)$ \\
\hline Vočadlo & $\tau=\left[\tau_{0}^{l / n}+\mu \dot{\gamma}\right]^{n}$ & & $(3.42)$ \\
\hline Casson & $\tau=\left[\tau_{0}^{1 / n}+(\mu \dot{\gamma})^{1 / n}\right]^{n}$ & & $(3.43)$ \\
\hline Shulman & $\tau=\left[\tau_{0}^{l / n}+(\mu \dot{\gamma})^{1 / m}\right]^{n}$ & & $(3.44)$ \\
\hline Briant & $\tau=\mu\left(1+\frac{\tau_{\infty}}{\kappa \mu \dot{\gamma}}\right)^{m} \dot{\gamma}$ & & $(3.45)$ \\
\hline Tscheuschner & $\tau=\left[\mu_{\infty}+\frac{\tau_{0}}{\dot{\gamma}}+\frac{\mu_{0}}{(\mu \dot{\gamma})^{n}}\right] \dot{\gamma}$ & & $(3.46)$ \\
\hline
\end{tabular}

It may be noted that the simplification of Shulman's model coefficients leads to the formulas describing the flow curves for simpler models of fluids such as:

- Casson's model: $m=n$ and $m=n=2$;

- Vočadlo's model: $m=1, n \neq 0$;

- Herschel's-Bulkley's model: $m \neq 0, \quad n=1$;

- Bingham's model: $m=n=1$;

- Ostwalda-de Wael's model: $n=1, \quad \tau_{0}=0$;

- Newton's model: $m=n=1, \quad \tau_{0}=0$.

\section{Summary and conclusions}

Despite the existence of many mathematical models describing the behavior of the aqueous humor in eye structures, which relatively well capture the essence of the phenomena in the eye, a common feature of these models should be noted; namely, the assignment of rheological properties of water to the aqueous humor. This simplification is probably due to the fact that the investigation of the rheological properties of the aqueous humor is impeded. Difficulties in the study of the rheological properties of the aqueous humor arise from the fact that it is a substance found in the body in very small quantities. In a normal eye there is only $3.000[\mu l]$. The amount that can be taken without disruption of the eyeball functioning is very small. A considerable increase in the level of complexity of the analytical model when taking into account the rheological properties of the aqueous humor is also of significant importance. The increased complexity of the model will complicate the obtainment of analytical solutions.

It is also important that biological fluids under extracorporeal conditions are subject to rapid degradation. Along with the degradation of the material all its characteristics change. Hence it is necessary to set the measurement path near the place where test material is taken (Chotard-Ghodsnia and Verdier, 2007; Fauci and Gueron, 2001; Verdier et al., 2009). 
In the paper, a proposal to use rheological models of Newtonian and non-Newtonian fluids (Walicka, 2002; Walicka and Walicki, 2012) for mathematical modeling of the aqueous humor flow in the structures of the eye is presented. Equations for the permeability of the porous material and liquid flow velocity in a porous medium are given. The trabecular meshwork was modeled as a porous medium, which is a rectilinear array of parallel capillary tubes. A Newtonian fluid, a couple-stress fluid and a generalized second grade fluid of a Shulman's type were considered as examples.

\section{Nomenclature}

$$
\begin{aligned}
& A_{O} \quad \text {-surface of the trabecular meshwork - in human body about } 18\left[\mathrm{~mm}^{2}\right] \\
& A_{v} \text { - contact surface of the vitreous and aqueous humor- in human body about } 65\left[\mathrm{~mm}^{2}\right] \\
& C_{p s} \text { - flow conductance of the aqueous humor- in human body about } 0.061-0.081\left[\frac{\frac{\mu l}{\mathrm{~min}}}{\mathrm{mmg}}\right] \\
& C_{t o t}, C_{p p} \quad-\text { total flow conductance }\left[\frac{\frac{\mu l}{\min }}{m m H g}\right]\left[\frac{\mu l}{\frac{s}{P a}}\right] \\
& C_{\text {trab }}, C_{T M} \text { - conductance flow through the trabecular meshwork - in human body about } 0.28\left[\frac{\frac{\mu l}{\min }}{\mathrm{mmHg}}\right] \text { or } 3.8 \cdot 10^{-5}\left[\frac{\frac{\mu l}{\mathrm{~s}}}{P a}\right] \\
& C_{u}-\text { flow conductance through the sclera- in human body about } 0.02\left[\frac{\frac{\mu l}{\min }}{\mathrm{mmHg}}\right] \\
& \text { F - geometric tensor } \\
& F \quad \text { - flow rate }\left[\frac{\mu l}{\min }\right] \\
& F_{f} \quad \text { - flow of the aqueous humor by filtration - about } 90 \% \quad F_{i n}\left[\frac{\mu l}{\min }\right] \\
& F_{i} \text { - total inflow of the aqueous humor to the eyeball, in human body about } 2-2.5\left[\frac{\mu l}{\min }\right] \\
& F_{o}-\text { total outflow of the aqueous humor from the eyeball }\left[\frac{\mu l}{\min }\right] \\
& F_{S}-\text { flow of the aqueous humor through active secretion- about } 10 \% F_{\text {in }}\left[\frac{\mu l}{\min }\right] \\
& F_{t} \text { - outflow of the aqueous humor through the trabecular meshwork }\left[\frac{\mu l}{\min }\right] \\
& F_{u} \quad \text {-outflow of the aqueous humor through the sclera } 6.7 \cdot 10^{-3}\left[\frac{\mu l}{s}\right] \text { or } 1.12 \cdot 10^{-4}\left[\frac{\mu l}{\min }\right] \\
& G \quad \text { - the shear modulus of the iris, set as } G=\frac{1}{3} E=9[\mathrm{kPa}] \\
& p \text { - pressure }[\mathrm{mmHg}] \\
& p_{e}, p_{v} \text { - episcleral vessels pressure - in human body about 7.6-11.6 }[\mathrm{mmHg}] \\
& p_{i}, p, \bar{p}-\text { intraocular pressure- in human body about } 15.6 \pm 3.2[\mathrm{mmHg}]
\end{aligned}
$$




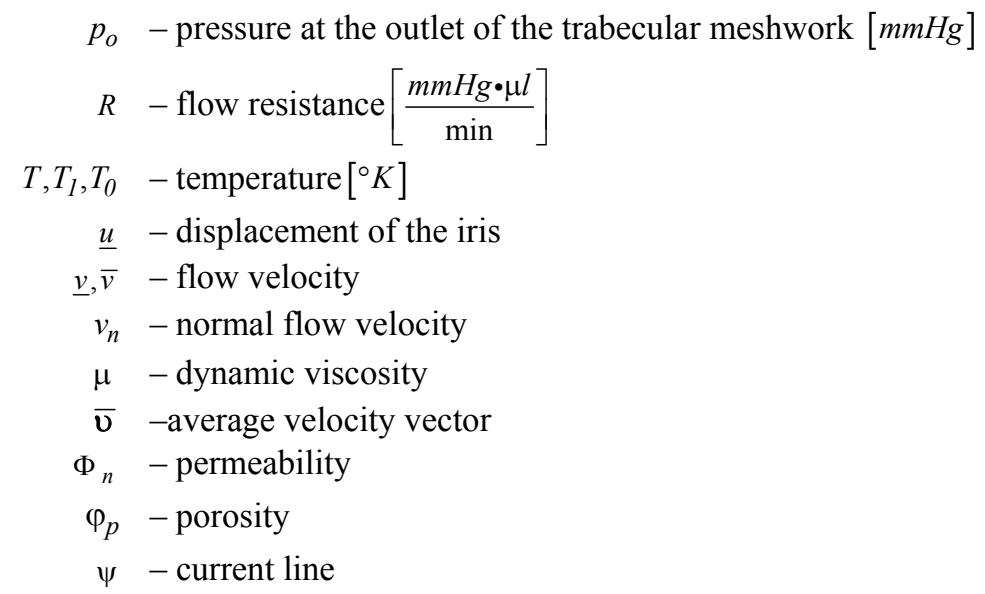

\section{References}

Amiri A. and Vafai K. (1994): Analysis of dispersion effects and non- thermal equilibrium, non-Darcian variable porosity incompressible flow through porous media. - Int. J. Heat and Mass Transf., vol.37, pp.939-954.

Amiri A. and Vafai K. (1998): Transient analysis of incompressible flow through a packed bad. - Int. J. Heat and Mass Transf., vol.41, pp.4259-4279.

Chotard-Ghodsnia R. and Verdier C. (2007): Rheology of living materials. Chapter in Rheology of Living Materials. pp.1-31. DOI: 10.1007/978-0-8176-4411-6_1.

Fauci L.J. and Gueron S. (2001): Computational Modeling in Biological Fluid Dynamics. - New York: Springer Science+Business Media.

Fitt A.D. and Gonzalez G. (2006): Fluid Mechanics of the Human Eye: Aqueous Humour Flow in the Anterior Chamber. - Bulletin of Mathematical Biology, DOI 10.1007/s11538-005-9015-2.

Gabelt B.T. and Kaufman P. (1995). Aqueous humor hydrodynamics. - In: Kaufman PL, Alm A, Ed. Alderes Physiology of the Eye, vol.8. St. Louis, MO: Mosby, pp.237-289.

Gedde S.J. (2012): The tube trials: new information to guide clinicians updates on six recent studies. - Ophthalmology Management, vol.12, No.16, pp.52-58.

Goldmann H. (1950) Minute volume of the aqueous in the anterior chamber of the human eye in normal state and in primary glaucoma. - Ophthalmologica. vol.120, No.1-2, pp.19-21.

Heys J.J., Barocas V.H. and Taravella M.J. (2002): Modeling passive mechanical interaction between aqueous humor and iris. - J. Biomech. Eng.., vol.123, No.6, pp.540-547.

Man C.S., Sun Q.X. (1987): On the significance of normal stress effects in the flow of glaciers. - J. Glaciology, vol.33, No.115, 268-273.

Millar C.J., Gabelt B. and Kaufman P. (2011): Aqueous Humor Dynamics. - Duane's Clinical Ophthalmology, Lippincott-Raven Publishers, Chapter 45, pp.1-34.

Nakayama A., Kuwahara F. and Hayashi T. (2004): Numerical model ling for three-dimensional heat and fluid flow through a bank of cylinders in yaw. - J. Fluid Mech., vol.498, pp.139-159.

Nakayama A., Kuwahara F. and Kodama Y. (2006): A thermal dispersion flux transport equation and its mathematical modeling for heat and fluid flow in a porous medium. - J. Fluid Mech. vol.563, pp.81-96.

Niżankowska M.H. (2006): Basic and Clinical Science Course. - Science 10, Glaucoma, Medical Publisher Urban and Partner, Wroclaw.

Stokes V.K. (1966): Couple-stresses in fluids. - Phys. Fluids, vol.9, No.9, pp.1709-1715. 
Tsai C.Y., Novack M. and Roffe G. (1988): Rheological and heat transfer characteristics of flowing coal-water mixtures. - Final Report, DOE/Mc 23255-2763.

Verdier C., Etienne J., Duperray A. and Preziosi L. (2009): Review. Rheological properties of biological materials. - C. R. Physique, Special Issue on "Complex and Biofluids", Ed. C. Misbah, vol.10, pp.790-811.

Walicka A. (2012): Porous curvilinear squeeze film bearing with rough surfaces lubricated by a power-law fluid. Journal of Porous Media, vol.15, No.1, pp.29-49.

Walicka A. (2002): Rheodynamics of Non-Newtonian Fluids Flows in Straight and Curved Channels (in Polish). Zielona Góra: University Press.

Walicka A. and Walicki E (2012): Rheodynamics of flows in porous biomaterials. - Mechanics in Medicine, vol.11, pp.181-193.

Walicka A. and Walicki E. (1999): Inertia effect in the squeeze film of a couple-stress fluid in biological bearings. Applied Mechanics and Engineering, vol.4, No.2, pp.363-373.

Walicki E. and Walicka A. (1998): Flow of generalized second grade fluids in a circular pipe. - Les Cahiers de Rhéologie, vol.34, No.1, pp.317-324.

Received: August 23, 2015

Revised: October 3, 2015 